\title{
Bioanalysis
}

\section{Bioanalytical inspections: organizational changes and regulatory perspectives}

\author{
"The Division of Bioequivalence and GLP Compliance, responsible \\ for CDER's Bioavailablity/Bioequivalence Inspection Program and the \\ Good Laboratory Practice Inspection Program, became the Office of \\ Study Integrity and Surveillance."
}

Keywords: batch acceptance $\bullet$ biosimilars $\bullet$ extract stability $\bullet$ immunogenicity testing - regulatory $\bullet$ US FDA

First draft submitted: 3 March 2016; Accepted for publication: 17 March 2016; Published online: 15 April 2016

During January 2015, several offices within the Center for Drug Evaluation and Research (CDER) were re-organized to improve consistency of decision-making, and to streamline policy, procedures and communications. Some Divisions were elevated to Office status because of the increased number of personnel resulting from the passage of the Generic Drug User Fee Amendments of 2012 (GDUFA). The resources provided by the new user fees supported hiring more personnel to address the significantly increased workload and newly imposed timelines. The Division of Bioequivalence and GLP Compliance (DBGLPC), responsible for CDER's Bioavailablity/Bioequivalence (BA/BE) Inspection Program and the Good Laboratory Practice (GLP) Inspection Program, became the Office of Study Integrity and Surveillance (OSIS). Additionally, OSIS became the newest suboffice under CDER's Office of Translational Sciences. Previously, this group was in the Office of Scientific Investigations under CDER's Office of Compliance. OSIS currently includes the Division of Generic Drug Bioequivalence Evaluation conducting and reviewing work under the BA/BE Inspection Program, and the Division of New Drug Bioequivalence Evaluation, which, in addition to the $\mathrm{BA} / \mathrm{BE}$ program, also conducts and reviews inspections under the GLP Inspection Program. Concurrent with the reorganization, OSIS began to implement a surveillance approach to inspections in conjunction with the application-based inspections, conducted previously.

This commentary provides an overview of changes in procedures within OSIS; it will also briefly discuss topics of interest that came up during the past year, including assays related to therapeutic biologics and immunogenicity testing.

\section{Procedural changes}

Prior to January 2015, scientific reviewers in CDER's Office of Generic Drugs or in the Office of Clinical Pharmacology usually determined the need for inspections during their reviews of studies supporting drug applications submitted to the Center. If an inspection was deemed necessary, a written consult requesting an inspection was generated by the review division and submitted to DBGLPC. DBGLPC prepared assignment memoranda for inspections of the clinical components of BA/BE studies and sent them to the Office of Regulatory Affairs (ORA) to conduct the inspections. The memoranda provided the background information for the inspection. If the inspection request was for the analytical component of a BE study, the same procedure was followed; however, analytical inspections included participation by DBGLPC staff to provide any necessary scientific expertise.

Beginning in January 2015, the new office (OSIS) began implementing a surveillance

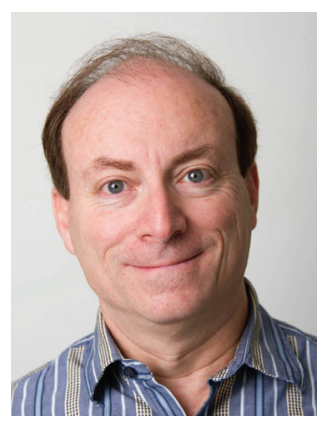

Sam H Haidar

Author for correspondence: US Food \& Drug Administration, 10903 New Hampshire Ave., Silver Spring, MD 20993, USA

sam.haidar@fda.hhs.gov

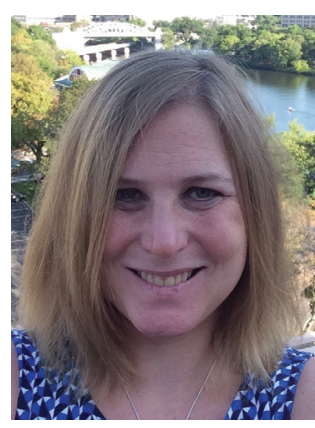

Kara A Scheibner

US Food \& Drug Administration, 10903 New Hampshire Ave., Silver Spring, MD 20993, USA 
approach to inspections. Surveillance is conducted contemporaneously with study-directed or application-based inspections, requested by the review divisions. As a result, OSIS is now responsible for selecting sites for routine inspections of clinical and bioanalytical sites that conduct studies for submitted applications. Currently, project management and members of the Collaboration, Risk-Evaluation and Surveillance Team (CREST) within OSIS evaluate sites in incoming applications to determine the need for inspections based on site history and other factors. The objective is to optimize resources and streamline procedures commensurate with CDER's significant increase of obligations under GDUFA.

\section{"Beginning in January 2015, the new office (OSIS) began implementing a surveillance approach to inspections."}

Under surveillance inspections, greater emphasis is placed on collecting information about overall site performance. Inspectors document the knowledge and experience of personnel, site procedures, commitment to good scientific principles and adherence to regulations. Inspection procedures include evaluating relevant studies, in addition to data audits and thorough review of individual studies. The information collected about the site plays a significant role in determining the timing and frequency of future inspections.

In addition to the organizational changes during 2015, OSIS conducted several inspections in support of therapeutic biologics applications to CDER, including studies supporting biosimilars. Inspection and evaluation of immunogenicity studies presented a new set of challenges. This was reflected by the difficulties some sponsors experienced while conducting regulated studies. As a result, CDER identified some studies requiring extensive review and the potential need for significant modification. This topic is discussed in greater detail further in this article. Topics related to analytical methods of small molecules were also examined within the office, when inspections encountered multiple approaches to similar types of testing. Two examples, extract stability and the inclusion of quality control samples in extraction batches, are described further in the following sections.

\section{Processed sample (extract) stability}

Testing conducted on samples postextraction (other than concentrations) can serve different objectives or address different concerns. Examples include: when a run is interrupted due to instrument failure or other reasons; when a run is stored in the autosampler for a period of time prior to injection; when processed samples are stored at room temperature or in the refrigerator; stability of samples at the beginning of a run compared with those at the end of the run; and finally, reinjection reproducibility where a whole run may be injected twice and variability in the results is determined. Different terminology has been ascribed to the testing listed above [1,2]. For example, the US FDA's Guidance for Industry: Bioanalytical Method Validation, published in 2001 refers to testing conducted postextraction as postpreparative stability [3]. Others have used terms such as processed sample stability, extract stability, autosampler stability and processed sample integrity. This has led to some confusion in industry, especially because some testing may require the use of freshly prepared standard curves or calibrators (e.g., storing extracted samples for a period of time), and some do not (e.g., re-injection reproducibility). In addition to the experience we have gained through inspections, OSIS staff has participated in efforts to identify the various approaches for testing processed samples in an effort to determine best practices, and provide better recommendations. Efforts in this regard are ongoing.

Several factors should be considered when conducting stability testing on extracted samples. As described in the 2009 White Paper on Recent Issues in Regulated Bioanalysis from the 3rd Calibration and Validation Group Workshop, there is always the possibility of similar degradation between the internal standard and the analyte of interest [1]. Simply reinjecting samples after storage could provide misleading results. For example, the ratio between the internal standard and the analyte may be the same, although significant degradation may have taken place. To address this concern, freshly prepared calibrators may be used. Another approach, which may be used when samples are left in the autosampler for a period of time, involves injection of a run the first time, waiting for a given number of hours, injecting the samples a second time, then determining concentration based on the results from the calibrators in the first run. As such, stability concerns would be detected without the need for using freshly prepared calibrators. Other factors to consider include evaporation and possible chemical reactions resulting from prolonged exposure to air (oxygen). This may occur after sample vial membranes are punctured by the sampling needle following the first injection of the run. Excessive evaporation can be expected when the reconstitution solution has a large component of organic solvent(s).

\section{Acceptance of processed batches}

Next we consider the topic of run acceptance in cases of runs comprising two or more individually (sepa- 
rately) processed (extracted) batches. The European Medicines Agency (EMA) Guideline on bioanalytical method validation recommends the following: "In case a whole run comprises more batches, acceptance criteria should be applied to the whole run and to the individual batches. The run can be acceptable, although a batch might have to be rejected, as criteria were not met" [4]. As such, QCs at three different concentrations (low, medium and high) are to be included in each batch. This is consistent with FDA's position. Practical or technical limitations notwithstanding, FDA currently recommends the inclusion of low, medium and high QCs in duplicates in individually processed batches. If singlet QCs are utilized at the three concentrations, then failure of any of the QCs within the batch could cast doubt on the accuracy of the unknowns within that batch, even if the run as a whole passes. Then, the batch may need to be repeated. This will be determined on a case-by-case basis.

\section{Biologics \& biosimilars}

In 2015, review and evaluation of a large number of biologics applications, including those supporting biosimilars, revealed specific areas of scientific concern within immunogenicity studies. The following sections highlight some of the critical observations OSIS has made over the past year while inspecting antidrug antibody (ADA) and neutralizing antibody (NAb) assays in support of immunogenicity assessments for therapeutic proteins.

\section{Selection of a relevant low positive control antibody}

Selection of a positive control antibody for use in $\mathrm{ADA} /$ immunogenicity testing can be challenging. Available affinity-purified antibodies are not necessarily representative of antibodies produced in response to a therapeutic protein, and results obtained are potentially the 'best case scenario.' Thus, it is important that assay values of selected positive control concentrations are representative of antibody levels present in study samples. This is especially critical for the low positive control (LPC); the LPC should adequately monitor performance of the ADA screening assay at response levels surrounding the screening assay cut point. The language in the FDA draft guidance, "Assay Development for Immunogenicity Testing of Therapeutic Proteins" [5] is that the selected LPC concentration should result in an assay rejection rate of $1 \%$ (i.e., the LPC assay signal should fall below the cut point $1 \%$ of the time).

During recent inspections of biologics applications with immunogenicity testing, we have observed instances of selected LPC concentrations that yield an assay response significantly higher than the screening cut point; in some studies the LPC assay response has been up to tenfold greater. Conversely, study sample assay response values have been very close to the screening assay cut point, and therefore significantly less than the LPC response. Using an LPC concentration with a high signal response compared with the cut point prevents adequate monitoring of assay performance and precision of assay values around the cut point. Consequently, the precision of study sample assay values that fall in the low range of the assay cannot be confirmed, and there is little confidence in the ability of the assay to distinguish potentially ADA-positive versus potentially ADA-negative samples in the screening assay. Relevance of the LPC is also an important consideration in the confirmatory assay. If the assay signal of the LPC is significantly higher than the screening cut point, consistent signal inhibition in the confirmatory assay does not accurately reflect potential study samples with a signal response in the low range of the assay. Thus, demonstration of consistent assay signal inhibition (above the confirmatory cut point) at an LPC concentration yielding assay values close to the screening cut point is critical to show the ability of the assay to positively detect ADAs in all study samples.

\section{Reproducibility between Tier 1 \& Tier 2 assays}

During inspection of studies, we have observed study samples that screen positive in the Tier 1 assay, but do not 'screen positive' in the Tier 2 assay (i.e., the unspiked study samples within a confirmatory assay have assay response values below the screening cut point). The result of this scenario is a degree of uncertainty in the reliability of the assay: if study samples screen positive in Tier 1 and negative in Tier 2, there is a distinct possibility that the converse scenario could occur (i.e., study samples that screened negative in Tier 1 could test positive in Tier 2). Thus, in these studies, the assay appears to have significant reproducibility concerns, and potentially positive samples may be missed in the initial screening (Tier 1) assay.

In addition, this scenario poses a question of how to handle samples that screen positive in Tier 1, 'screen' negative in Tier 2 and confirm positive in Tier 2. We have seen examples of such samples reported as positive, placing confidence in the highly specific confirmatory assay. However, there are also examples of such samples reported as negative; justification for reporting the sample as negative was based on the fact that a sample which screened negative in the confirmatory assay cannot be confirmed positive, thus placing greater confidence in the screening assay.

These inconsistencies present several questions that should be discussed and addressed within the immu- 
nogenicity testing community: should there be an expectation for reproducibility of unspiked study samples in ADA assays as an indicator of a reliable assay? Should there be a consensus among firms in how these data are reported? If yes, does scientific rationale favor the confirmatory assay or the screening assay? Should such study results trigger repeat analysis? And, is there an underlying cause of assay nonreproducibility that should be considered?

\section{Interference in cell-based neutralizing antibody assays}

Using a cell-based assay format to determine presence of neutralizing antibodies in study samples is highly desirable because of the functional relevance of the assay. However, cell-based assays also add additional layers of complexity and variability that must be addressed during method validation. One such additional layer is interference from endogenous molecules (e.g., cytokines) present in control serum samples and serum from study samples. Assessment of potential interference from these molecules is critical to ensuring reliability of the assay, and prevention of false positive or negative results.

In assessing the potential for interference in a NAb assay, choice of cell type is highly important. The ability of a cell to respond to both the therapeutic protein of choice (e.g., erythropoietin), and to other potential stimulating molecules, should factor into the decision of which cells are best suited for a NAb assay. If the cell line chosen for the assay responds to multiple stimuli, the specific concentration level at which interference in the assay will be present should be determined for each molecule. This knowledge should subsequently be applied when testing the study samples. Assessing the level of potentially interfering endogenous molecules is critical in all study samples, for example, via an ELISA assay. This is particularly true in patient populations where levels of these molecules can be elevated due to

\section{References}

1 Savoie N, Garofolo F, van Amsterdam P et al. 2009 White Paper on Recent Issues in Regulated Bioanalysis from The 3rd Calibration and Validation Group Workshop. Bioanalysis 2(1), 53-68 (2010).

2 Nowatzke W, Woolf E. Best practices during bioanalytical method validation for the characterization of assay reagents and the evaluation of analyte stability in assay standards, quality controls, and study samples. AAPS J. 9(2), E117E122 (2007).

3 US Food and Drug Administration. Guidance for Industry: Bioanalytical Method Validation. US Department of Health a specific disease. If levels of interfering molecules cannot be assessed, an alternative method should be used to determine whether study samples contain molecules that could potentially stimulate cells in the absence of study drug. Finally, if endogenous molecules are present in specific study samples at concentration levels that will interfere with the assay, a method to negate this interference should be assessed and validated. For example, high levels of endogenous cytokines can often be removed using monoclonal antibody depletion protocols prior to study sample analysis in the NAb assay.

\section{Conclusion}

The topics of discussion presented herein represent a small subset of the processes, methodology and scientific rationale that are critical to traditional $\mathrm{BE}$ studies, and to bioanalysis of biologics and biosimilars. Further information on these and other topics is available in the manuscripts and guidances referenced in this commentary.

\section{Disclaimer}

The views expressed in this article do not necessarily represent the official views or policies of the US FDA.

\section{Acknowledgements}

The authors wish to thank William (Bill) Taylor and Sean Kassim for their insightful comments and edits of this Commentary.

\section{Financial \& competing interests disclosure}

The authors have no relevant affiliations or financial involvement with any organization or entity with a financial interest in or financial conflict with the subject matter or materials discussed in the manuscript. This includes employment, consultancies, honoraria, stock ownership or options, expert testimony, grants or patents received or pending, or royalties.

No writing assistance was utilized in the production of this manuscript.

and Human Services, US FDA, Center for Drug Evaluation and Research, MD, USA (2001)

www.fda.gov/downloads/Drugs/Guidances/ucm070107.pdf

4 European Medicines Agency. Guideline on bioanalytical method validation. EMEA, Committee for Proprietary Medicinal Products (CPMP), London, UK (2011). www.ema.europa.eu

5 US Food and Drug Administration. Guidance for Industry: Assay Development for Immunogenicity Testing of Therapeutic Proteins (Draft). US Department of Health and Human Services, US FDA, Center for Drug Evaluation and Research, MD, USA (2009). www.fda.gov 